\title{
Challenges in the Pharmacologic Management of Obesity and Secondary Dyslipidemia in Children and Adolescents
}

\author{
Mary Jayne Kennedy $\cdot$ Kevin D. Jellerson • \\ Michael Z. Snow $•$ Michelle L. Zacchetti
}

Published online: 16 May 2013

(C) The Author(s) 2013. This article is published with open access at Springerlink.com

\begin{abstract}
The rise in childhood obesity has lead to an increased number of children with lipid abnormalities and the predominance of a combined dyslipidemic pattern characterized by a moderate-to-severe elevation in triglycerides, normal-to-mild mild elevation in LDL cholesterol and reduced HDL cholesterol. Although recently published National Heart, Lung and Blood Institute (NHLBI) guidelines represent a significant step forward in managing primary dyslipidemias in pediatric patients, there is still no general consensus regarding the pharmacologic treatment of obesity-related lipid abnormalities in children. The use of early pharmacologic intervention to control dyslipidemias and reduce cardiovascular risk in young children is only expected to increase given the steady increase in obesity and emergence of atherosclerotic disease in pre-adolescents. Despite the increasing use of lipidlowering therapy in children over the last few years, diet and lifestyle modification remain the first line therapy. Given the challenges of instituting and maintaining lifestyle modification in pediatric patients, however, it is likely that institution of drug therapy may be required in many children. Of all the medications currently available, the fibric acid derivatives have a cholesterol lowering profile that is most likely to be effective in obese children with the high TG/low HDL phenotype and data from a recently published study of gemfibrozil in children with metabolic syndrome are promising. However, additional information
\end{abstract}

M. J. Kennedy $(\varangle)$ · K. D. Jellerson · M. Z. Snow ·

M. L. Zacchetti

Department of Pharmacotherapy and Outcomes Science,

School of Pharmacy, Virginia Commonwealth University,

410 N. 12th Street, PO Box 980533, Richmond,

VA 23298-0533, USA

e-mail: mjkennedy@vcu.edu regarding the short and long-term safety and efficacy of fibrate therapy in children with obesity-related lipid disorders is needed before use of these agents can be recommended.

\section{Introduction}

Coronary heart disease (CHD) is one of the leading causes of mortality in the United States accounting for approximately 1 in 6 deaths each year [1]. Although the clinical manifestations of CHD are most apparent in adults, there is increasing evidence to suggest that the atherosclerotic process begins during early childhood. Fatty streaks, the earliest subclinical manifestations of atherosclerosis, have been noted in the coronary arteries of children as young as 2 years of age and the prevalence in children ages 2 to 15 years is estimated to be $50 \%$ [2]. The extent of atherosclerotic lesions in children and young adults has also been significantly correlated with traditional cardiovascular risk factors (dyslipidemia, hypertension, obesity) and increases with the number of factors present [2].

The number of children and adolescents with risk factors for cardiovascular disease in the United States continues to increase at an unprecedented rate in large part due to the significant increase in childhood obesity. Over the past 3 decades, the prevalence of obesity in children ages 6 to 11 years has increased from $4 \%$ to over $20 \%$ [1]. Among children ages 2 to 19 years, approximately 23.5 million (32\%) are overweight and 12 million (17\%) are obese [1]. As in adults, there is a strong association between childhood obesity and the clustering of multiple cardiovascular risk factors including insulin resistance, dyslipidemia and elevated blood pressure, a combination known in adults as the metabolic syndrome [3]. This clustering of obesity 
related risk factors continues into adult life and is strongly associated with an accelerated atherosclerotic process. Weight gain, insulin resistance and an atherogenic lipid profile are also well-described side effects of psychotropic drugs such as the second-generation antipsychotics now widely used for a variety of conditions in pediatric patients [4].

With the increased prevalence of obesity in children, secondary causes have superseded primary etiologies of cardiovascular risk in pediatric patients. As a result of this evolving phenotypic profile, the therapeutic targets and recommendations outlined in standard consensus guidelines for the management of major cardiovascular risk factors in children (which focus on risk factors of primary etiologies) do not apply to a majority of at-risk children. This is particularly true for the management of pediatric dyslipidemias. The increase in pediatric obesity rates has lead to the predominance of a combined dyslipidemic pattern in children that is characterized by a moderate-to-severe elevation in triglyceride (TG) level, normal-to-mild mild elevation in LDL cholesterol and a reduced HDL cholesterol level [3]. When the original consensus guidelines were developed by the National Cholesterol Education Program (NCEP) of the National Heart, Lung and Blood Institute (NHLBI) in 1992, however, the focus was almost exclusively on identification and management of children with an elevated LDL cholesterol level-a pattern most frequently associated with dyslipidemias of primary or familial origin [5].

In late 2011, a set of integrated guidelines for cardiovascular health and risk reduction in children and adolescents, which includes updated recommendations for identifying and managing dyslipidemias, was released by NHLBI and endorsed by the American Academy of Pediatrics (AAP) [3]. The updated NHLBI guidelines, although reflective of the changing dyslipidemia phenotype in children, do not provide clear recommendations for management of children less than 10 years of age with concomitant risk factors $\left(\mathrm{BMI} \geq 95^{\text {th }}\right.$ percentile, evidence of $\mathrm{CHD}$ ) or complex mixed dyslipidemias that are associated with obesity. The guidelines also highlight the lack of age-specific information regarding use of cholesterol lowering therapies such as the fibrates, that specifically target TG elevations commonly seen in obese children. The use of early pharmacologic intervention to control dyslipidemias and reduce cardiovascular risk in young children is only expected to increase, however, given the steady increase in obesity and emergence of atherosclerotic disease in pre-adolescents.

\section{Therapeutic Options for the Treatment of Secondary Dyslipidemias in Children}

There are several classes of medications available to treat lipid abnormalities in pediatric patients. A majority of children with secondary lipid abnormalities will have a moderate-to-severe elevation in TG levels, normal-to-mild mild elevation in LDL cholesterol and a reduced HDL cholesterol level. Given that the individual phenotypic profile will differ from patient to patient, however, fasting lipid profile results should ultimately be used to guide treatment selection.

\subsection{HMG-CoA Reductase Inhibitors}

The most common medications used to treat lipid abnormalities in children and adults are members of the HMGCoA reductase inhibitor, or statin class. Statins inhibit 3-hydroxy-3-methyl-glutaryl coenzyme, an endogenous reductase involved in the rate-limiting step of cholesterol synthesis. This action results in lower intracellular cholesterol levels and up-regulation of LDL receptors leading to increased clearance of LDL from the circulation. The statins are very effective in reducing levels of LDL cholesterol and have been reported to lower levels by 20-50\% [6]. Adverse effects associated with statin use in adults include increased hepatic transaminase levels, elevations in creatine kinase, rhabdomyolysis (rare) and potential teratogenicity.

HMG-CoA reductase inhibitors are the most studied class of lipid lowering drugs in the pediatric population and are the preferred pharmacologic therapy for lowering LDL cholesterol in children and adolescents [3]. The extent of LDL reduction achieved in children with statin therapy is comparable to that observed in adults and ranges from 21 to $39 \%$ depending on the dose and specific agent used [7]. Improvements in vascular endothelial function and carotid artery intima media thickness (IMT), both markers of early atherosclerosis, have also been observed in children ages 8 to 18 years after approximately 2 years of statin treatment [8, 9]. Several longitudinal studies have also demonstrated that, as a class, statins do not negatively impact growth, development or sexual maturation when used in children ages 8 to 18 years for an average duration of up to 4.5 years [10, 11]. However, there is still concern that inhibition of cholesterol production during a critical period of growth and maturation may have adverse consequences, particularly in children that are pre-pubertal. Clinical experience with the statins in children less than 8 years of age therefore remains limited.

There are currently seven FDA-approved HMG-CoA reductase inhibitors; six of the seven have a pediatric indication for reducing LDL cholesterol in children with familial hypercholesterolemia. Pravastatin $(20 \mathrm{mg})$ is the only statin indicated for use in children ages 8 years and older; lovastatin, simvastatin, fluvastatin, atorvastatin, pravastatin (40 $\mathrm{mg}$ ) and rosuvastatin are approved for use in children ages 10 years and older. It is important to note 
that labeling of statins for pediatric use was based solely on demonstration of acceptable safety and efficacy in children with familial and other monogenic forms of hypercholesterolemia. Given the lack of comparable data in children with secondary dyslipidemias, recommendations for the use of statins in this patient population have been extrapolated from data in children with an entirely different disease etiology. However, the dose-exposureresponse relationship for statins is likely different for each dyslipidemic phenotype making the validity of extrapolation between two disease etiologies somewhat questionable.

\subsection{Cholesterol Absorption Inhibitors}

The cholesterol absorption inhibitors are a relatively new class of medications that inhibit the uptake of dietary and biliary cholesterol in the intestines most likely via blockade of the Niemann-Pick C1-like 1 protein that mediates intestinal cholesterol absorption. Reduced cholesterol absorption results in decreased cholesterol delivery to the liver. As a result, LDL receptor expression is upregulated and LDL cholesterol clearance is increased. Given that ezetimibe selectively inhibits cholesterol absorption, TG and fat-soluble vitamin levels are not affected. The cholesterol absorption inhibitors have been associated with few adverse effects other than gastrointestinal discomfort since they act locally in the gastrointestinal tract. However, systemic effects are possible. Ezetimibe is the only medication in this class currently on the market and it is available alone $\left(\right.$ Zetia $\left.^{\circledR}\right)$ and in combination with the HMG-CoA reductase inhibitor simvastatin $\left(\right.$ Vytorin $\left.{ }^{\circledR}\right)$. Although ezetimibe effectively lowers LDL cholesterol when used both alone and in combination with a statin in adults, its clinical benefit on cardiovascular risk reduction has yet to be proven [12].

The safety and efficacy of ezetimibe in children have been evaluated in a limited number of short-term studies [13-15]. When used as monotherapy for up to 16 months in children as young as 5 years of age, ezetimibe effectively lowers total cholesterol and LDL-cholesterol without causing any significant side effects $[13,14]$. In adolescents with familial hypercholesterolemia (ages 10-17 years), coadministration of ezetimibe with simvastatin for up to 53 weeks provides greater LDL cholesterol reduction compared with simvastatin alone [15]. Ezetimibe is currently approved for the treatment of familial hypercholesterolemia in children 10 years of age and older. Given its favorable adverse effect profile and availability as a small tablet formulation, ezetimibe has the potential to become an important new therapy for the treatment of elevated LDL cholesterol in young children. It is likely that ezetimibe will be used predominantly in children with persistent elevations in LDL cholesterol after treatment with other medications has failed. However, its precise role in the treatment of primary and secondary dyslipidemias in children remains undefined.

\subsection{Fibric Acid Derivatives}

Fibric acid derivatives (gemfibrozil, fenofibrate) are a class of drugs traditionally used to treat hypertriglyceridemia in adults. The mechanism of action of this therapeutic class is complex and incompletely understood. It is thought that fibric acid derivatives decrease hepatic production of TG by increasing oxidation of fatty acids in muscle and the liver and reducing the rate of hepatic lipogenesis. These agents are also believed to increase HDL cholesterol through a complex mechanism regulated by the nuclear transcription activator, peroxisome proliferator-activated receptor- $\alpha$ [16]. The fibric acid derivatives primarily affect TGs (reduced levels) and HDL cholesterol (increased levels). The effect on LDL cholesterol is variable. In adults, the predominant side effects are gastrointestinal upset and abdominal pain. Increased liver function tests (LFTs) have also been reported. Although uncommon, myopathy and rhabdomyolysis may occur particularly when these agents are used in combination with a statin.

The fibric acid derivatives are useful in the treatment of adult patients with combined dyslipidemias. Limited published experience in children also suggests that these agents may be effective in treating the obesity-related high TG/low HDL cholesterol phenotype [17-20]. Use of fibric acid derivatives for up to 6 months in children as young as 4 years of age has been shown to effectively lower total cholesterol, LDL-cholesterol and TG levels and increase HDL levels. Treatment was well tolerated although some moderate increases in LFTs were noted [17-19]. The fibric acid derivative, gemfibrozil, has also recently been shown to effectively lower TG levels (54\% decrease) and increase HDL cholesterol levels (17\% increase) in children with metabolic syndrome when used in conjunction with diet and exercise [20]. The drug was well tolerated over the 8-month treatment period and only 2 of 47 children reported muscle pain. At present, use of the fibric acid derivatives is limited primarily to children with severe elevations in TGs $(\geq 500 \mathrm{mg} / \mathrm{dL})$ who are at risk for pancreatitis [3]. Although these agents currently do not have FDA approved labeling for use in children, collective experience from off-label use in young children suggests that a reassessment of the role of fibric acid derivatives in the treatment of obesity-related dyslipidemias may be warranted. 


\subsection{Bile Acid Binding Resins}

Bile acid binding resins (cholestyramine, colestipol, colesevelam), also known as bile acid sequestrants, act by binding bile salts within the intestinal lumen and preventing their reuptake in the terminal ileum. This leads to a depletion of bile salts and increased conversion of cholesterol to bile in the liver. Decreased intracellular levels of cholesterol in the hepatocytes also leads to upregulation of LDL cholesterol receptor activity and increased clearance of LDL from the circulation [21]. The bile acid binding resins may increase TG levels and interfere with the absorption of fat-soluble vitamins, particularly vitamins A, $\mathrm{D}$ and $\mathrm{K}$. They also have a high potential for drug interactions since they act as an anion-absorbing resin in the gut. These agents are not systemically absorbed but are associated with significant gastrointestinal side effects (including constipation, bloating and flatulence) that are often treatment limiting.

Although never approved for use in children, bile acid binding resins were long considered to be the only suitable lipid lowering therapy in children and only recently have been supplanted by statins as the preferred drug treatment for increased LDL cholesterol in pediatric patients. Studies of bile acid sequestrants in children and adolescents aged 6 to 18 years with elevated LDL cholesterol levels (131 to $190 \mathrm{mg} / \mathrm{dL}$ ) have resulted in total cholesterol reductions of $7-17 \%$ and LDL cholesterol reductions of 10-20\% sometimes with a modest elevation in TG level [3]. As in adults, adverse gastrointestinal effects are common in children and compliance is frequently poor with both the tablet and granular powder formulations with discontinuation rates ranging from 20 to $30 \%$ [22]. Recently, a second generation bile acid binding resin with enhanced binding capacity for bile acids (colesevelam), was shown to effectively reduce LDL cholesterol alone and in combination with a statin in children with familial hypercholesterolemia with a relatively low incidence of gastrointestinal side effects $(10 \%)$ and high compliance rate (over $85 \%$ ) [23]. Further studies are needed however, to better delineate the role of this novel bile acid binding resin in the management of pediatric lipid disorders.

\subsection{Niacin}

Niacin is a hydrophilic B complex vitamin that reduces LDL cholesterol and TG levels and increases HDL cholesterol by decreasing the hepatic production and release of very low-density lipoprotein (VLDL). It is also the only medication known to reduce lipoprotein(a) levels. Niacin has a very favorable lipid lowering profile, particularly for patients with the high TG/low HDL phenotype, but its use is limited by poor tolerance associated with its significant adverse effects. The most common side effects of niacin therapy are flushing, hepatic failure, myopathy, glucose intolerance and hyperuricemia that can be severe and treatment limiting.

To date, there has only one observational study performed in children $(n=21$, ages 4 to 14 years $)$ treated with niacin (500 to $2250 \mathrm{mg} /$ day) [24]. In this study, children received treatment for an average duration of 8 months and while statistically significant reductions in total and LDL cholesterol were noted, there was no change in TG or HDL cholesterol levels. The drug was also very poorly tolerated with $71 \%$ of children experiencing flushing, $26 \%$ experiencing elevation in hepatic transaminase concentrations and $38 \%$ discontinuing therapy due to adverse effects. Due to its poor tolerance, potential for serious adverse effects and lack of safety and efficacy data in pediatric patients niacin therapy is not routinely recommended for treatment of pediatric dyslipidemias and its use is reserved for children with homozygous familial hypercholesterolemia and/or elevated lipoprotein(a) levels.

\section{$2.6 \omega-3$ Fatty Acids}

The $\omega-3$ fatty acids, eicosapentaenoic acid (EPA) and docosahexaenoic acid (DHA), have been studied extensively for their ability to lower TG levels in adults. In adults, $\omega-3$ fatty acids have been shown to lower TG levels by $30-40 \%$ and raise HDL cholesterol by 6 to $17 \%$ when administered in doses of 2 to 4 grams per day [3]. There is also compelling evidence that $\omega-3$ fatty acids can reduce the risk of coronary artery disease when used in conjunction with dietary modification [25]. Although their exact mechanism of action remains unknown, the $\omega$ - 3 fatty acids are thought to decrease hepatic fatty acid and TG synthesis while simultaneously increasing fatty acid degradation and oxidation leading to reduced VLDL cholesterol release [3]. In addition to their effects on TG, $\omega-3$ fatty acids also increase HDL cholesterol and decrease LDL cholesterol particle size.

$\omega-3$ fatty acids are not synthesized de novo in humans to a great extent and fish is the major dietary source of EPA and DHA. The American Heart Association (AHA) recommends that adults with coronary artery disease consume about 1 gram of EPA + DHA per day and that adults with hypertriglyceridemia consume 2 to 4 grams of EPA + DHA per day [25]. The United States Government has not established a recommended daily intake amount of $\omega-3$ fatty acids in children although the typical pediatric diet is generally deficient of $\omega-3$ fatty acids [26]. There are many dietary supplements that contain EPA and DHA currently on the market but these products are not regulated by the FDA and are highly variable with respect to the content of $\omega-3$ fatty acids and other ingredients. Lovaza ${ }^{\circledR}$, 
the only prescription form of $\omega-3$ fatty acids currently available, is a highly concentrated formulation of the ethyl esters of EPA and DHA and is approved as an adjunct to dietary modification for the treatment of adults with hypertriglyceridemia ( $\mathrm{TG} \geq 500 \mathrm{mg} / \mathrm{dL}$ ).

There have been no studies of Lovaza ${ }^{\circledR}$ in pediatric patients and experience with $\omega-3$ fatty acids in children is limited to a small number of studies conducted with various dietary supplement preparations. The EARLY study was a 6-month, randomized double-blind, placebo-controlled cross-over study conducted in 20 children in which the effect of DHA on lipoprotein subclasses was evaluated [27]. Although no significant quantitative changes in total cholesterol, LDL cholesterol, HDL cholesterol or TG were reported, there was a significant decrease in low-density lipoprotein subclasses 1 and 3 and high density lipoprotein 2. Treatment with DHA supplementation was well tolerated and there were no reported adverse effects. In children and adolescents (ages 11 to 21 years) with familial hyperlipoproteinemia, a commercially available preparation of fish oil containing $18 \%$ EPA, $12 \%$ other $\omega$-3 fatty acids and vitamin $\mathrm{E}$ also had no effect on lipid parameters but was associated with epistaxis (8/11 subjects) and prolongations of bleeding time (3/11 subjects) [28].

While concerning, the majority of clinical evidence in adults does not support an increased risk of bleeding with high-dose fish oil even when used in combination with anticoagulant or antiplatelet therapy [29]. It is important to note, however, that because of developmental differences in the dose-exposure-response relationship children may have an increased predisposition to certain adverse effects such as bleeding. Care must therefore be taken when extrapolating clinical experience in adults to the pediatric population. Given the positive effects on TG and HDL, $\omega-3$ fatty acids may be useful in the treatment of children with the obesity-related dyslipidemic phenotype either alone or in combination with niacin or a fibric acid derivative. However, additional data are needed before definitive recommendations regarding the use of $\omega-3$ fatty acids in children can be made.

\section{Overview of Updated NHLBI Expert Panel Recommendations}

\subsection{Assessment of Lipid and Lipoprotein Levels in Children and Adolescents}

Preliminary evidence in children with familial hypercholesterolemia suggests that early identification and control of dyslipidemia substantially reduces accelerated early atherosclerosis (as measured via carotid intima media thickness) and future cardiovascular disease risk in pediatric patients [8-11]. However, the age at which screening for dyslipidemia should be initiated and the type of screening approach (universal or targeted) remain somewhat controversial. Multiple prospective screening studies have mapped the normal distribution of lipids and lipoproteins throughout childhood and have demonstrated a clear developmental pattern characterized by a decrease in total and LDL cholesterol (10-20\%) during puberty [3]. On the basis of this developmental pattern, the NHLBI guidelines recommend universal screening in all children between the ages of 9 and 11 years (Grade B recommendation) [3]. Targeted screening is also recommended in children ages 2 to 8 years in children with one or more of the following conditions: diabetes, hypertension, BMI $\geq 95^{\text {th }}$ percentile, a moderate to high risk medical condition, positive history of smoking or family history associated with increased cardiovascular risk (Grade B recommendation) [3]. While this strategy will likely identify a majority of children with obesity-related dyslipidemias, it creates a clinical dilemma in that there are currently no definitive recommendations for treating lipid abnormalities in children less than 10 years of age.

The NHLBI guidelines delineate specific cut points for acceptable, borderline-high and high plasma lipid, lipoprotein and apolipoprotein concentrations in children and adolescents and recommendations for initiation and modification of treatment are based on these cut points [3]. The fasting lipid profile is the recommended method of screening and decisions regarding the need for medication therapy should be based on the average of results from at least 2 profiles obtained at least 2 weeks but more than 3 months apart (Grade B recommendation). It is important to note that most dyslipidemias associated with obesity do not meet current cutoffs for pharmacologic treatment particularly with respect to LDL cholesterol levels. Given that even moderate elevations in cholesterol are associated with increased cardiovascular risk, however, it raises the question as to whether current cut points are appropriate for children with the obesity-related dyslipidemia phenotype.

\subsection{Drug Therapy Selection for Children with the High TG/Low HDL Lipid Phenotype}

Non-pharmacologic approaches remain the cornerstone of treatment for lipid abnormalities in children. The first step proposed for managing children with elevated levels of LDL cholesterol $(\geq 130-250 \mathrm{mg} / \mathrm{dL})$ or TG $(\geq 100$ $\mathrm{mg} / \mathrm{dL}-500 \mathrm{mg} / \mathrm{dL}$ in children $<10$ years; $\geq 130-500 \mathrm{mg} / \mathrm{dL}$ in children 10-19 years) is a focused intervention on diet and lifestyle modification for at least 3 to 6 months (Grade $\mathrm{A}$ and $\mathrm{B}$ recommendation) [3]. In children with elevated TG levels, reduction of simple carbohydrate intake and weight loss can effectively reduce TGs. When associated 
with obesity, decreased caloric intake and increased activity levels are also of primary importance. Unfortunately, however, diet and lifestyle modification can be difficult in practice and many times, social and family circumstances can lead to relapses. This is particularly true for children who are often not in control of their food selections/choices.

The NHLBI guidelines recommend that children younger than 10 years of age should not be treated with a medication unless they have severe primary hyperlipidemia or a high-risk condition that is associated with serious medical morbidity (Grade C recommendation) [3]. Screening recommendations, however, suggest that fasting lipid profiles be performed in children ages 2 to 8 years with diabetes, hypertension, $\mathrm{BMI} \geq 95^{\text {th }}$ percentile, a moderate to high risk medical condition, positive history of smoking or family history associated with increased cardiovascular risk. It is therefore highly likely that children who fall outside of the scope of the current treatment recommendations but who have clinically significant lipid abnormalities will be identified via this targeted screening approach.

In children aged 10 to 21 years, the primary goal of therapy is to control LDL cholesterol. In children with normal LDL cholesterol who are less than 10 years of age with TG levels between 100 and $200 \mathrm{mg} / \mathrm{dL}$ or between 10 to 19 years with TG levels between 130 and $200 \mathrm{mg} / \mathrm{dL}$ an increase in dietary fish intake, in conjunction with continued diet and lifestyle modification, is recommended (Grade $\mathrm{D}$ recommendation). If TG levels are between 200 and $499 \mathrm{mg} / \mathrm{dL}$, then treatment with $\omega-3$ fatty acids may be considered (Grade D recommendation). If non-HDL cholesterol is also $\geq 145 \mathrm{mg} / \mathrm{dL}$ then treatment with a statin, fibrate and/or niacin can also be considered in consultation with a lipid specialist (Grade D recommendation). Currently there are no specific recommendations for increasing HDL cholesterol in this age group.

\section{Challenges in the Pharmacotherapy of Children with Secondary Hyperlipidemia}

There are many challenges associated with the treatment of lipid abnormalities in children and these challenges have become even greater with the shift towards an obesityrelated dyslipidemia phenotype. While children with familial hypercholesterolemia comprise the largest group of pediatric patients with significantly elevated LDL cholesterol, obese children with the high TG/low HDL phenotype currently comprise the majority of children with lipid abnormalities. Most clinical experience in treating lipid abnormalities in children, however, has been derived from children with biochemical alterations in LDL cholesterol and may not be applicable to children with other forms of dyslipidemia. In adults, niacin and the fibric acid derivatives are the most effective agents in lowering TG and increasing HDL cholesterol levels. Of all the cholesterol lowering therapies, these agents are the least well tolerated, are associated with the highest rates of treatment non-compliance and have the least experience in pediatric patients.

While it may be possible to extrapolate information regarding the efficacy of and/or response to cholesterol lowering agents from adults to children, developmental differences in drug absorption, distribution, metabolism, and elimination make it difficult to develop pediatric dosing recommendations using pharmacokinetic data generated in adults. This relative lack of age-specific information regarding the dose-exposure response relationship for most cholesterol lowering medications in young children and adolescents makes dose selection and titration particularly challenging. There is also limited information regarding the safety of administering cholesterol lowering medications to pre-pubertal children and there is some concern that exposure to these medications could potentially affect cholesterol dependent production of steroid hormones in the gonads and adrenal glands [30]. Although data from short-term studies suggest that cholesterol-lowering therapy does not adversely affect the processes of growth and maturation, there have been no long-term studies evaluating the potential implications of exposure to these medications during critical periods of development. This has resulted in a general reluctance to develop drug therapy guidelines for children less than 10 years of age despite the recommendation that children ages 2 to 8 years with risk factors (including obesity) be screened for lipid abnormalities.

Although attempts have been made to mathematically model the best time to initiate lipid-lowering therapy the optimal age at which treatment should be initiated remains unknown [31]. Irrespective of when therapy is initiated, however, it is likely that treatment with cholesterol-lowering medications will be life-long resulting in a cumulative level of drug exposure that far exceeds that in adults. Nevertheless, studies of lipid-lowering therapy in children to date have all been relatively short term (less than 5 years) and the safety of long-term exposure to these medications remains unknown. Because long-term followup studies have not been completed, the impact of cholesterol lowering therapy on reducing adverse cardiovascular outcomes that often do not manifest until adulthood is unclear. Carotid intima-media thickness may be a good surrogate marker of treatment response in this patient population although it may be years before the clinical benefit of pharmacologic therapy can be documented using this strategy. The ethics of prescribing life-long 
cholesterol-lowering therapy without a known risk-reduction benefit is therefore an important consideration. The potential psychological effects of long-term cholesterol lowering treatment, such as being embarrassed or stigmatized, is an additional unique pediatric consideration that must also be recognized.

\section{Summary and Conclusions}

The dramatic rise in childhood obesity has led to an increased number of children with lipid abnormalities that are phenotypically different from those that were most prevalent when the first comprehensive intervention guidelines were published in 1992 [5]. The recently-published NHLBI consensus guidelines, which recognize the changing dyslipidemia phenotype and incorporate a decision tree for the pharmacologic management of increased $\mathrm{TG}$, represent a significant step forward in the management of this continually evolving condition [3]. However, there is still no general consensus regarding the pharmacologic treatment of obesity-related lipid abnormalities that are present in the vast majority of children. Studies to date evaluating the safety and efficacy of lipid lowering therapy in children with secondary hyperlipidemia have been limited and primarily focused on lowering LDL cholesterol. Of all the medications currently available, the fibric acid derivatives have a cholesterol lowering profile that is most likely to be effective in obese children with the high TG/ low HDL phenotype and recently published data with gemfibrozil in children with metabolic syndrome are promising [20]. However, additional information regarding the short and long-term safety and efficacy of fibrate therapy in children with obesity-related lipid disorders is needed.

Acknowledgements Drs. MJK, KDJ, MZS and MLZ have declared no conflicts of interest that are relevant to the contents of this review. No external funding was used in the preparation of this review.

Open Access This article is distributed under the terms of the Creative Commons Attribution Noncommercial License which permits any noncommercial use, distribution, and reproduction in any medium, provided the original author(s) and the source are credited. The exclusive right to any commercial use of the article is with Springer.

\section{References}

1. Roger VL, Go AS, Lloyd-Jones DM, et al. Executive summary: heart disease and stroke statistics-2012 update. A report from the American Heart Association. Circulation. 2012;125:188-97.

2. Berenson GS, Srinivasan SR, Bao W, Newman WP, Tracy RE, Wattigney WA. Association between multiple cardiovascular risk factors and atherosclerosis in children and young adults. N Engl J Med. 1998;338:1650-6.

3. Expert Panel on Integrated Guidelines for Cardiovascular Health and Risk Reduction in Children and Adolescents; National Heart, Lung and Blood Institute. Expert Panel on Integrated Guidelines for Cardiovascular Health and Risk Reduction in Children and Adolescents: Summary Report. Pediatrics 2011;128(Supplement 5):S213-56.

4. Correll CU. Monitoring and management of antipsychotic-related metabolic and endocrine adverse events in pediatric patients. Int Rev Psychiatry. 2008;20:195-201.

5. National Cholesterol Education Program (NCEP): highlights of the report of the expert panel on blood cholesterol levels in children and adolescents. Pediatrics 1992;89:495-501.

6. Waters DD. What the statin trials have taught us. Am J Cardiol. 2006;98:129-34.

7. Avis HJ, Vissers MN, Stein EA, et al. A systematic review and metaanalysis of statin therapy in children with familial hypercholesterolemia. Arterioscler Thromb Vasc Biol. 2007;27:1803-10.

8. Wiegman A, Hutten BA, de Groot E, et al. Efficacy and safety of statin therapy in children with familial hypercholesterolemia. A randomized controlled trial. JAMA. 2004;292:331-7.

9. de Jongh S, Lilien MR, op't Roodt J, Stroes ESG, Bakker HD, Kastelein JJP. Early statin therapy restores endothelial function in children with familial hypercholesterolemia. J Am Coll Cardiol. 2002;40:2117-21.

10. Carreau V, Girardet JP, Bruckert E. Long-term follow-up of statin treatment in a cohort of children with familial hypercholesterolemia. Efficacy and tolerability. Pediatr Drugs. 2011;13:267-75.

11. Rodenburg J, Vissers MN, Wiegman A, et al. Statin treatment in children with familial hypercholesterolemia. The younger the better. Circulation. 2007;116:664-8.

12. Kastelein JJ, Akdim F, Stroes ES, et al. Simvastatin with or without ezetimibe in familial hypercholesterolemia. N Engl J Med. 2008;358:1431-43.

13. Yeste D, Chacon P, Clemente M, Albisu MA, Gussinye M, Carrascosa A. Ezetimibe as monotherapy in the treatment of hypercholesterolemia in children and adolescents. J Pediatr Endocrinol Metab. 2009;22:487-92.

14. Clauss S, Wai KM, Kavey REW, Kuehl K. Ezetimibe treatment of pediatric patients with hypercholesterolemia. J Pediatr. 2009;154:869-72.

15. van der Graaf A, Cuffie-Jackson C, Vissers MN, et al. Efficacy and safety of coadministration of ezetimibe and simvastatin in adolescents with familial hypercholesterolemia. J Am Coll Cardiol. 2008;52:1421-9.

16. Auwerx J, Schoonjams K, Fruchart JC, Staels B. Transcriptional control of triglyceride metabolism: fibrates and fatty acids change the expression of the LPL and apo C-III genes by activating the nuclear receptor PPAR. Atherosclerosis. 1996;124:S29-37.

17. Steinmetz J, Morin C, Panek E, Siest G, Drouin P. Biological variations in hyperlipidemic children and adolescents treated with fenofibrate. Clinica Chemica Acta. 1981;112:43-53.

18. Becker M, Staab D, Von Bergmann K. Long-term treatment of severe familial hypercholesterolemia in children: effect of sitosterol and bezafibrate. Pediatrics. 1992;89:138-42.

19. Wheeler KAH, West RJ, Lloyd JK, Barley J. Double blind trial of bezafibrate in familial hypercholesterolemia. Arch Dis Child. 1985;60:34-47.

20. Smalley CM, Goldberg SJ. A pilot study in the efficacy and safety of gemfibrozil in a pediatric population. $\mathrm{J}$ Clin Lipidol. 2008;2:106-11.

21. Shepherd J, Packard CJ, Bicker S, et al. Cholestyramine promotes receptor-mediated low-density-lipoprotein catabolism. N Engl J Med. 1980;302:1219-22. 
22. McCrindle BW, O'Neill MB, Cullen-Dean G, Helden E. Acceptability and compliance with two forms of cholestyramine in the treatment of hypercholesterolemia in children: a randomized, crossover trial. J Pediatr. 1997;130:266-73.

23. Stein EA, Marais AD, Szamosi T, et al. Colesevelam hydrochloride: efficacy and safety in pediatric subjects with heterozygous familial hypercholesterolemia. J Pediatr. 2010;156:231-6.

24. Colletti RB, Neufeld EJ, Roff NK, McAuliffe TL, Baker AL, Newburger JW. Niacin treatment of hypercholesterolemia in children. Pediatrics. 1993;92:78-82.

25. Kris-Etherton PM, Harris WS, Appel LJ. Fish consumption, fish oil, omega-3 fatty acids and cardiovascular disease. Circulation. 2002;106:2747-57.

26. Devaney B, Ziegler P, Pac S, Karwe V, Barr SI. Nutrient intakes of infants and toddlers. J Am Diet Assoc. 2004;104:S14-21.
27. Engler MM, Engler MB, Malloy MJ, Paul SM, Kulkarni KR, Mietus-Snyder ML. Effect of docosahexaenoic acid on lipoprotein subclasses in hyperlipidemic children (the EARLY study). Am J Cardiol. 2005;95:869-71.

28. Clarke JT, Cullen-Dean G, Regelink E, Chan L, Rose V. Increased incidence of epistaxis in adolescents with familial hypercholesterolemia treated with fish oil. J Pediatr. 1990;116: 139-41.

29. Bays HE. Safety considerations with omega-3 fatty acid therapy. Am J Cardiol. 2007;99:35C-43C.

30. Bhatnagar D. Should pediatric patients with hyperlipidemia receive drug therapy? Pediatr Drugs. 2002;4:223-30.

31. Ulrich S, Hingorani AD, Martin J, et al. What is the optimal age for starting lipid lowering treatment? A mathematical model. BMJ. 2000;320:1134-40. 